\title{
Women with ovarian cancer during childbearing years felt that they looked normal, but "in [their] skin, [they were] different"
}

\author{
Schaefer KM, Crago Ladd E, Lammers SE, et al. In your skin you are different: women living with ovarian cancer during
} childbearing years. Qual Health Res 1999 Mar;9:227-42.

QUESTION: What is the experience of women living with ovarian cancer during their childbearing years?

\section{Design}

Hermeneutic phenomenology.

\section{Setting}

Lehigh Valley, Pennsylvania, USA.

\section{Patients}

5 women who had been diagnosed with ovarian cancer 1-10 years before the study were recruited from a private practice.

\section{Methods}

Each woman participated in 3-4 indepth interviews, which lasted 60-90 minutes and continued until both the researcher and participant agreed that there was no more to say. Thematic analysis was guided by Van Manen's method. To ensure trustworthiness of findings, the researchers kept reflective journals, checked the analyses with participants, and resolved differences in interpretation of meaning through discussion.

\section{Main findings}

The complexity of the experience of living with cancer during the childbearing years was revealed in 12 themes. Serendipitous diagnosis of ovarian cancer occurred in the context of infertility workups, painful periods, or prenatal care. Women had to be persistent in obtaining appointments and second opinions that ultimately led to a diagnosis of cancer. They usually were told of the diagnosis (and of the requisite hysterectomy) when they were recovering from anaesthesia; most did not remember being aware of the diagnosis until several days later. Managing treatment was a challenge early on because women felt healthy and did not yet feel the impact of the diagnosis. Feeling ill as a result of chemotherapy made it difficult to believe that treatment was making them better. Women were disconcerted with the extent of losses they experienced. The horrible hair experience was not only hair loss but also total body baldness, which reminded them of the disease and the treatment.

The abrupt and premature loss of childbearing capacity led to feelings of violation of [women's] sense of being and resentment that the choice of having a hysterectomy or having another child was taken away from them. They were angry that they could not undertake the expected cultural narrative of having children. Women talked about the unfairness of menopause, as they were unprepared for the symptoms, life pattern disruptions, and body changes that accompanied menopause. They questioned whether symptoms were a result of menopause, cancer, or chemotherapy.

Women expressed a sense of being normal and being different. Their need to do "normal" things and be "normal" people took place in the context of physical changes, fatigue, and changes in their perception of self. Being different involved both not being the type of woman expected to get cancer (being at risk) and changes in the personal self as a result of the cancer.
Other themes were intimate dreaming (revelation of feelings in dreams); the importance of being with others; feelings of not being heard by physicians; being vigilant in attending to clues that the cancer might be returning; and going through a process of comprehending or seeking meaning in their illness.

\section{Conclusion}

Women living with ovarian cancer during their childbearing years experienced a sense of being different; differences came about as a result of the diagnosis, hysterectomy and the resultant onset of early menopause and loss of childbearing capacity, chemotherapy, and the search for meaning in their experiences.

\section{COMMENTARY}

This powerful study by Schaefer et al provides readers with an intimate description of the needs, concerns, and meanings that women attach to the experience of living through ovarian cancer during their childbearing years.

This phenomenological study has addressed an absence in the literature and has allowed the voices of women who live with ovarian cancer to be heard. The data generated in the interviews with 5 women were rich and concise and served to sensitise the reader to the experience of living through ovarian cancer.

The decision trail of both the process and method of the study was clearly articulated. Skilfully weaving the relevant literature in with the women's words provides the reader with an understanding of how the researchers arrived at their findings.

12 themes emerged that illuminated the fears, sorrows, and joys of the women's journeys with ovarian cancer. As a consequence of their cancer experience the women felt different although, deceptively, they outwardly appeared the same. The women sought meaning from their experience with cancer and the overwhelming loss associated with being unable to bear children. The meaning of living through ovarian cancer for these women was not simply about living with the symptoms of illness but had an impact on every aspect of their life. It seems important for health professionals to provide opportunities for women to communicate openly about their illness experiences. In doing so, a foundation of acceptance and respect for the whole person may be established, which will give women permission to ask questions, seek assistance, and be a partner in their health care on an ongoing basis. The acknowledgment of a woman's experiences by a health professional is affirming for women who are struggling with the unfamiliar effects of illness. Through this study, readers are sensitised to the complexity of the experiences of women living through ovarian cancer during the childbearing years, and, as such, the findings are of importance to nurses and to the wider healthcare community.

Debbie Kralik RN, MN, MRCNA Research Associate Royal District Nursing Service Glenside, South Australia 